\title{
Challenges of Future R\&D in Mobile Communications
}

\author{
Dr. Anwar M. Mousa \\ University of Palestine-Gaza- Palestine \\ Tel +970599609918 Fax +972828 80006
}

\begin{abstract}
This paper provides a survey for the main challenges of future research and development $(\mathrm{R} \& D)$ for next generation mobile networks (NGNs). It addresses software and hardware reconfigurability with focus on reconfigurable coupling and interworking amongst heterogeneous wireless access networks. It also explores the promising technologies for NGNs such as nanotechnology, cloud computing and texting by thinking. The paper then highlights the challenging research areas for enhancing network performance and affordability beginning with All IP network and security issues, Vehicular Ad Hoc Networks (VANET) and the necessity of high data rates at cell edges. Investigating the viability of cooperative networks and one unified global standard for NGNs, the paper analyzes macrodiversity and advanced multi-cell coordination for mitigating inter-cell interference. Direct device-to-device communication, global coverage using satellites and high-altitude platforms are presented as possible evolution paths for space roaming and extending NGN' radio-access. Finally, the paper inspects the most developing applications for NGNs such as World-Wide Wireless Web (WWWW), machine-type communication, wireless sensor networks (WSNs), e-healthcare systems and wearable devices with AI capabilities.
\end{abstract}

Keywords- R\&D; NGNs; re-configurability; VANET; nanotechnology; cloud computing; HAP; WWWW; WSNs; ehealthcare; wearable devices.

\section{INTRODUCTION}

The key concepts of next generation mobile networks (NGN) suggested in research papers are:

- Full interworking (interoperability) of heterogeneous systems.

- Up to $1 \mathrm{Gbit} / \mathrm{s}$ of broadband services, delivered by standard/system, for users in any environment [1].

- Real wireless world with no limit in access and zone.

- Wearable devices with AI capabilities.

- IPv6, where the assignment of the visiting care of mobile-IP address is done according to location and connected network [2].

- One unified global standard.

- Cognitive Radio (CR) based technology [3].

- High-altitude platforms station systems

- Wireless sensor systems for surveillance and environmental sensing.

For the time being, the 4-Generation (4G) systems are still under intensive research and development efforts; there are numerous research groups and development projects worldwide aiming at widespread $4 \mathrm{G}$ wireless and cellular deployments all over the world. Surely, researches will continue progressing in the future for beyond 4-G systems. This paper highlights main areas of research and development (R\&D) challenges that face NGN.

The paper is organized as follows: Section II discusses in details re-configurability in mobile communications with focus on re-configurability enablers and reconfigurable interworking amongst heterogeneous wireless access networks. Reconfigurable (adaptive) coupling of heterogeneous networks is proposed as new coupling method that adaptively changes coupling level from open, loose to tight and even very tight according to networks' load status and delay constraints. Hardware and software reconfigurability with different reconfiguration and downloading modes are also presented in section II. Section III explores the promising technologies for NGNs such as nanotechnology, cloud computing and texting by thinking. Network energy efficiency with reduced energy consumption necessary for both mobile terminal and radio access network is thoroughly illustrated. Section IV highlights the challenging research areas for enhancing network performance and affordability beginning with All IP network and security issues, Vehicular Ad Hoc Networks (VANET) and the necessity to high data rates at cell edges. It investigates the viability of cooperative networks and one unified global standard for NGNs. Macro-diversity and advanced multi-cell coordination for mitigating interference are also analyzed. Finally direct device-to-device communication and global coverage using satellites, and high-altitude platforms are presented at the end of section IV. Section V focuses on the most developing applications for NGNs such as World-wide wireless web (WWWW), machine-type communication, wireless sensor networks (WSNs), E-Healthcare Systems and wearable devices with AI capabilities. Section VI concludes the paper.

\section{RE-CONFIGURABILITY In MOBILE COMMUNICATIONS}

Re-configurability in mobile communication systems makes use of the abilities of reconfigurable terminal and networks for self-adaptation to a dynamically-changing environment aiming at improving service offering and spectrum utilization. To meet the rapidly increasing demand for mobile services, with the limited radio spectrum, more flexible ways to share radio spectrum among multiple services and radio networks are required. Re-configurability is based 
on Cognitive Radio (CR) which in turn is considered as an evolution of Software-Defined Radio (SDR) [4]. A CR is the final point of SDR platform evolution: a fully software and/or hardware reconfigurable radio that changes its communication functions depending on network and/or user demands. A CR is an SDR that additionally senses and tracks changes in its environment and then reacts upon its findings. The implementation of re-configurability in mobile systems spans from reconfigurable interworking (interoperability) among heterogeneous wireless access networks to reconfigurable (Adaptive) coupling of different networks. Re-configurability could be in both software and hardware. The reconfigurable software radio concept has been an active research and development topic for many years. With the power of SDR, network is dynamically reconfigurable; the programs running on the reconfigurable processing elements as well as the communication links between the processing elements are configured at run-time. Different downloading modes in software reconfiguration exist each having its own advantages and disadvantages; mission-oriented re-configurability and self- re-configurability. Hardware re-configurability is based on the evolution of semiconductor technology and mainly performed by operators; adding additional equipments to increase network capacity at a specific time. More illustration in the following subsections:

\section{A. Re-configurability enablers}

\section{1) Software Defined Radio}

Software Defined Radio (SDR) [5] benefits from today's high processing power to develop multi-mode (multiband and multi-standard) base stations and terminals. In future the terminals will adapt the air interface to the available radio access technology, however at present this is done by the infrastructure. Several infrastructure gains are expected from SDR. For example, to increase network capacity at a specific time (e.g. during festivals or sport events), an operator can reconfigure its network by adding several modems at a given Base Transceiver Station (BTS). SDR makes this reconfiguration easy. For the expected next generation systems, SDR will become an enabler for terminal and network re-configurability through software download. Figure 1 shows a general reconfigurable SDR transceiver in both terminal and base stations. It can be reconfigured via a control bus supplying the processing units with the parameters downloaded from remote re-configuration database via a predefined download channel. For manufacturer, this can be a powerful aid for providing multi-mode equipment with reduced development effort and costs.

Refer Figure 1 in the Appendix: General SDR transceiver

\section{2) Cognitive Radio}

Cognitive radio (CR) technology allows different radio access technologies to share the same spectrum efficiently. This is done by adaptively finding unused spectrum and reconfiguring (adapting) the transmission scheme to the requirements of the technologies currently sharing the spectrum. The applicability of CR to cellular communication is a relatively new area and further studies are required to assess the feasibility and impact of such usage. CR technologies includes the ability of equipments to determine their location, sense spectrum used by neighboring equipments, change their frequencies, adjust output power, and even alter transmission parameters. It is a transceiver that is able to understand (think) and react to its operating environment. Thus CR concerns both mobile terminal and networks which are computationally intelligent about radio resources and related communications. The Radio is aware about changes in its environment and responds to these changes by adapting operating characteristics in some way to improve its performance.

A CR technology for spectrum re-uses and network overlay, named Vandermonde-subspace frequency division multiplexing (VFDM) [6] is newly developed. It is a modulation scheme to allow the co-existence of macro-cells and cognitive radio small-cells in mobile network. VFDM, a technique for interference cancellation in overlay networks, allows a secondary network to operate simultaneously with a primary network on the same frequency band. It can be applied to block transmission systems with a guard time over frequency selective channels. It achieves zero interference towards the primary system by employing a specific pre-coder that aligns the data to the null space of the interfering channel from the secondary to the primary system.

\section{B. Reconfigurable Interworking amongst heterogeneous wireless access networks}

A seamless interworking amongst heterogeneous network represents the corner stone for the success of next generation systems with different evolving access technologies. A novel solution that ensures interworking between several types of wireless access network is given by the IEEE 802.21 standard [7]. The IEEE 802.21 is focused on handover implementation between different wireless networks in heterogeneous environments regardless of the type of medium. The standard names this type of vertical handover as Media Independent Handover (MIH). The goal of IEEE 802.21 is to facilitate the mobile nodes' usage by providing uninterrupted handover in heterogeneous networks. The heart of the 802.21 framework is the Media Independent Handover Function (MIHF) which is implemented in every IEEE 802.21 compatible device (in either hardware or software). This function is responsible for communication with different terminals, networks and remote MIHFs. It provides abstract services to the higher layers using a unified interface (L2.5 functionalities). Although the IEEE 802.21 standard is still in its formative stages, it may be the key enabler for seamless vertical handover and transparent roaming in heterogeneous networks. Hence, this standard will make a major contribution towards the reconfigurable interworking aspect of next generations wireless and cellular communications systems. The IST Ambient Networks projects [8], uses a single multi-radio system with powerful multi-radio resource management mechanisms and generic link layer functionality to integrate the envisioned future heterogeneous environment. This eases the provision of the "Always Best Connected" [9] approach paving the way towards interworking heterogeneous networks. Besides, the IST MAGNET project [10] and IST MAGNET Beyond project [11] provide a solution of some technological issues concerning interworking between different network interconnection schemes. 
The reconfigurable interworking can be implemented at the network level, the user level or both, bringing benefits from both the network providers' perspective and the users' perspective. It also contributes to the robustness of the provisioning of users' requested services allowing user seamless and transparent service management [12]. At the network level, the reconfigurable interworking allows network providers to select, with minimal investments, between alternative wireless access networks. The selection could be made based on several criteria such as:

- Comparison between the availability of radio access resources and service requirements (users' QoS requirements, channel state, outage probability, vertical handover probability...etc).

- Efficient load sharing between different coexisting wireless networks.

- Efficient spectrum sharing.

- Congestion control.

Thus, the changes in the network resource availability due to network instantaneous saturation can be bypassed by terminals and network components that are dynamically reconfigured (adapted) to the new situation. At the user level, the reconfigurable interworking of the heterogeneous systems leads to more efficient end-to-end connectivity and service delivery in heterogeneous environments. It also provides easier worldwide roaming and dynamic adaptation to regional contexts. The users' equipments reconfigure based on:

- Availability of spectrum and radio access resources

- Service cost Minimization when multiple technologies are available.

- Anticipation of user contexts and preferences.

\section{Reconfigurable (Adaptive) Coupling of Heterogeneous Networks.}

Different types of coupling of heterogeneous networks and therefore different integration approaches can be classified as follows:

\section{- $\quad$ Open coupling}

With open coupling, no effective integration exists between two or more radio access technologies with only a billing system being shared between them. Separate authentication procedures are used for each access networks and no vertical handovers take place. The only interaction happens between the billing management systems of each network technology, but no interaction exists between the control procedures related to the QoS and mobility management.

\section{- Loose coupling}

In loose coupling the operator is still able to use the existing subscriber database, allowing centralized billing and maintenance for different technologies. Besides, there is an interaction between the control planes of each operator regarding the authentication procedure. Hence, the user enjoys a unique subscription if the network provider is the same for both networks. The main disadvantage of loose coupling is that vertical handover is not seamless; during the handover between the two Radio Access Technologies (RATs), the service in progress is dropped. Besides, the handover delay is significant as mentioned above.

\section{- $\quad$ Tight coupling}

The advantage of tight coupling is that it permits seamless handover between radio access technologies to take place. However it requires the generic RAT networks to support necessary interfaces with other coupled technologies.

- Very tight coupling

In the very tight coupling, the interworking is provided within the radio access network. Due to a new interface definition between different RATs, the networks are able to perform a seamless inter-network handover. IST-TRUST project [13] focused a great effort on very tight coupling between networks and allowed for very fast vertical handover. Adaptive coupling is a new proposed mechanism that adaptively changes coupling level from open, loose to tight and even very tight according to networks' load status and delay constraints. On the one hand, if the integration between different technologies is tight, the provisioning of the service is more efficient and network selection as well as the vertical handover process is faster. However, a high level of integration requires considerable effort in the definition of interfaces and mechanisms able to support the necessary exchange of data and signaling between different radio access networks. Moreover, tight coupling suffers from potential of load congestion when one network full load is immersed on the other. On the other hand, if the integration between different technologies is loose, the delay of handover process is significant. On the positive side, loose coupling allows for the flexibility and independence of implementing individually different mechanisms within each network. Besides, it eases the gradual deployment of one network with no or little modification on the other network(s).

\section{Software Re-configurability}

Projects in literature dealing with software reconfigurability have focused on the technology for providing multi-mode software reconfigurable terminals. IST TRUST project [13] identified the frameworks and systems that are needed to support software reconfigurable radios from the users' perspective by examining user requirements. Realizing the user potential of reconfigurable radio systems, the project provided network connectivity and services when and where they are required. Hence, simultaneous connections to different RATs became possible. The IST MOBIVAS project [14] enabled flexible provisioning of value-added services in mobile communication networks by developing architectural approaches and prototypical implementations of integrated software platforms. The IST PACWOMAN project [15] enabled the design of low-cost and low-power reconfigurable user terminal by performing the necessary $R \& D$ on all OSI layers. Software reconfigurable transceivers, whether at the user terminal or at the base station, differ from a conventional transceiver only by the fact that they scan the available networks and, after downloading the appropriate software(s) reconfigure themselves as per the selected network features. 
Different reconfiguration and downloading modes exist as follows:

\section{1) Reconfiguration database download}

Software modules downloaded, via Internet, from a remote reconfiguration database using cloud computing. A transceiver can be reconfigured via a control bus supplying the processing units with the parameters which describe the desired standard. Such a reconfiguration guarantees that the transmission can be changed instantaneously if necessary; in case of inter-standard handover.

\section{2) Re-configurability using a dedicated channel}

A dedicated channel bridges between user terminal and base station facilitating error-free downloading of the requested software module. However this approach suffers from the varying bandwidth requirements over narrow-band channels and also of the cost of allocating a dedicated physical channel for downloading.

\section{3) Re-configurability using advanced SIM Cards}

Downloading and reconfiguring of the appropriate services on the terminals takes place upon insertion of smart cards including prior stored information regarding the reconfigurable software modules. However this approach is mostly suitable for stand-alone terminals.

\section{4) Re-configurability using predefined broadcasting pilot}

This approach is based on a predefined broadcasting global pilot and download channel (GPDCH) which is constantly monitored by multimode user terminals. On detection of any available network, the terminals decide whether to switch off or not. This approach suffers from long downloading timedue to slow speed of GPDCH.

\section{5) Mission-Oriented Re-configurability}

Reconfiguration to meet a given set of mission requirements is called mission oriented reconfiguration. Typical mission requirements might include operation within buildings, over long distances and while moving at high speed. Mission-oriented reconfiguration usually selects a set of radio software modules from a library of modules and connecting them into an operational radio.

\section{6) Self-Re-configurability}

A transceiver normally consists of several modules; a radio frequency front-end, a digital signal processor, and a control processor. In this approach, each module is a self-describing and the radio automatically reconfigures itself for operation from the available modules.

\section{E. Hardware Re-configurability}

Hardware reconfigurability cannot be separated from software reconfigurability. However the reconfigurable hardware is based on the evolution of semiconductor technology. More and more transistors can be integrated on a single chip which makes it possible to build large systems on a chip level, System-on-Chip (SoC) [16]. Hardware reconfigurability is made easy thanks to various processing elements such as General Purpose Processors (GPPs), Field Programmable Gate Arrays (FPGAs), Application Specific
Integrated Circuits (ASICs) and Domain Specific Reconfigurable Hardware (DSRH) modules. Different processing elements are used for different purposes. The GPPs are programmable to perform different computational tasks. FPGAs which are reconfigurable by nature, are efficient at performing bit-level operations but not for word level DSP operations. The ASICs are optimized for power and cost but cannot be reconfigured to adapt to new applications. The DSRH is a relatively new type of processing element, where the configurable hardware is adapted towards a specific application domain.

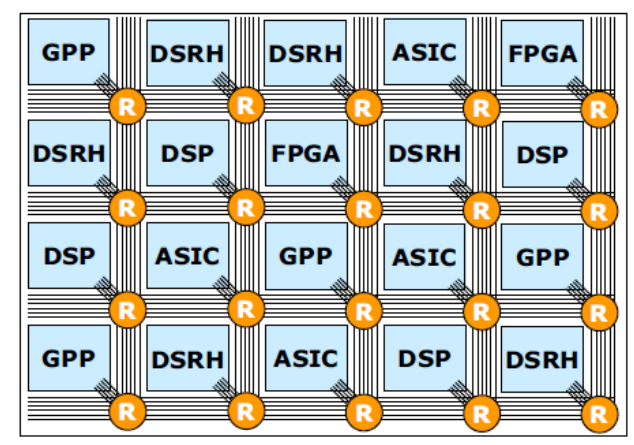

Figure 2: An example of a heterogeneous SoC. Source: from [16]

\section{PROMISING TECHNOLOGIES}

\section{A. Nanotechnology}

Nanotechnology is derived from nanometer scale; between 0.1 and $100 \mathrm{~nm}$. It uses nano-science to control specific processes. The field is also known as molecular nanotechnology (MNT) where MNT deals with control of the structure of matter based on atom-by-atom and molecule by molecule engineering. Nanotechnology is considered as the coming industrial revolution, and the communications industry will be radically transformed by it in a few years. Current technologies cannot resolve challenges such as more memory and computing power required to accommodate accelerating intelligence, more accurate sensing and increased data rates. Nanotechnology could provide effective solutions for more powerful computing, nano-sensing, memory enlargement, and human-machine interaction [17]. Nanotechnology will have considerable impacts on both mobile terminal as well as core network as follows:

- The mobile terminal has become more than a communication device in modern world; computation and communication are ready to serve the user in an intelligent way. Mobile terminals besides the intelligence embedded in human environments will enable ubiquitous sensing, computing, and communication. The Impact of nanotechnology over mobile terminals enables them to act as intelligent nano-enabled sensors. Nano-enabled sensors have applications in many industries, among them transportation, communications, medicine and safety. 
- The core network requires high speed and a reliable capacity to manipulate and interoperate the increasing number of heterogeneous access technologies. At present, nanotechnologies are used in Digital Signal Processing (DSP) Fabrication. Much more new perceptions are introduced in DSP designing which increases the overall system speed \& capacity.

Nanotechnology enables higher transfer rate of data within and between devices since a major limitation in transfer rate is the use of electrical wiring and contacts. Technologies under development that will lay the foundation for faster data transfer, more mobile processing power and larger data storage are:

- Nano-materials with novel optical, electrical, and magnetic properties.

- Faster and smaller non-silicon-based chipsets, memory, and processors.

- Computers based on Quantum Computing.

- Advanced manufacturing and microscopy systems.

- Smaller and faster optical switches.

- Nano-scale MEMS: micro-electro-mechanical systems.

\section{B. Network Energy Efficiency}

Reduced energy consumption is necessary for both mobile terminal and radio access network as follows:

\section{1) Mobile Terminal}

Low energy consumption for mobile terminals has been a critical concern since the emergence of hand-held terminals roughly 25 years ago. The driving force has been the reduction in battery size and improved battery time. A new nanotechnology called Power Felt is an innovative way to recharge battery by converting body heat into electric current. Hence the battery can be recharged at any time. Felt the power itself, made of carbon nano-tubes, is very small and locked in flexible plastic fibers that resemble fabric. This technology uses the difference between room temperature and the temperature of the body to create energy. Generally, thermoelectric is not yet fully developed technology to produce energy. Besides, the high cost of such technology makes it unfeasible to use in consumer products. Other promising method for recharging the mobile terminal is to be self powered; the phone derives its energy/power from the sun where it shines.

\section{2) Radio-Access Network}

Today, a close attention is paid to reduced energy consumption also in the radio-access network. This is due to the resultant global warming and increased energy consumption in the information and communication technology (ICT) industries. Energy efficiency has, hence, become a key performance metric to evaluate the performance of a communication network. To prevent our planet from global warming and effectively increase energy efficiency, it is expected that future wireless networks will use eco-friendly green energy. In some rural areas, it may not even be possible to connect the base station to the electrical grid. Instead of the diesel generators commonly used today, solar panels could be used as power source for low consumption. Moreover, the cost of energy is a considerable part of the overall operational cost for the operator. Nevertheless, the future evolution of cellular systems should further minimize unneeded transmission of signals by designing energy-aware routing algorithms with sustainable power models.

\section{Cloud computing}

Cloud computing [18] is a technology that uses the internet and central remote server to maintain data and applications. It is a model for enabling ubiquitous, on-demand access to a shared pool of reconfigurable computing resources such as servers, storage, applications, and services. In next generations networks this central remote server could be a content provider. Cloud computing allows consumers and business to use applications without installation and access their personal files at any computer with internet access. Cloud computing promotes service development where operators can enter the cloud computing market and create new value-added services by integrating industry content and applications in the digital supermarket model.

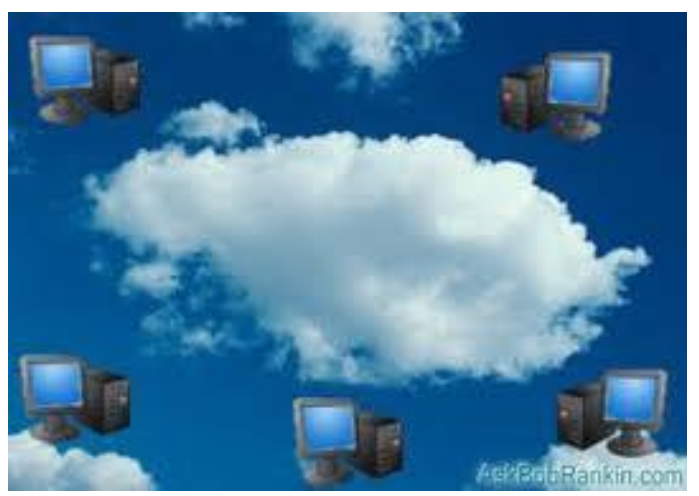

Figure 3: Cloud Computing, Ubiquitous Access. Source: from [19]

\section{Texting by thinking}

In future mobile generation, phones may become available with the option of texting by thought power alone. Even though one may think this is imaginative, it could be achieved by a sensor-mounted headset worn by the user. The device contains brain-machine interface technology which analyses brain waves, converts them into digital signals and displays the resulting letters on-screen. This allows completely handsfree texting, effectively creating a form of electronic telepathy. Even though the process is rather slow at this stage, nevertheless advances in the coming years will enable smooth and fast interactions, revolutionizing the world of communication.

\section{ENHANCED NETWORK PERFORMANCE AND AFFORDABILITY}

This section highlights the challenging research areas for enhancing network performance and affordability beginning with All IP network and security issues, Vehicular Ad Hoc Networks (VANET) and the necessity to high data rates at cell edges. It investigates the viability of cooperative networks and one unified global standard for NGNs. Macro-diversity and 
advanced multi-cell coordination for mitigating interference are also analyzed. Finally direct device-to-device communication and global coverage using satellites, and highaltitude platforms are presented.

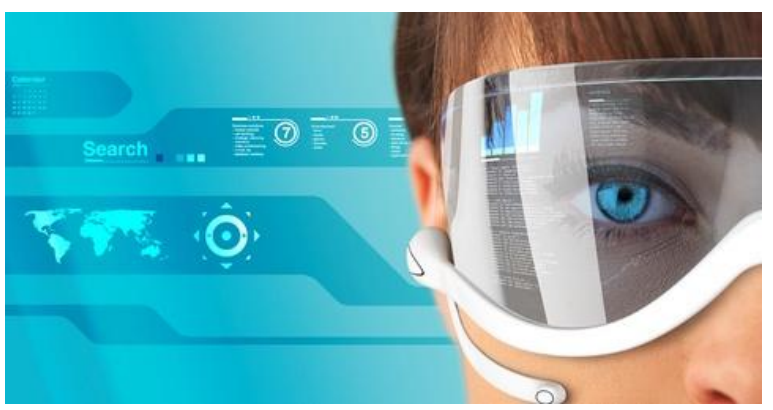

Figure 4: Texting by thinking, revolutionizing the world of communication.

\section{A. All IP Network and Security Issues}

The All-IP Network (AIPN) is an evolution of the 3GPP system to meet the increasing demands of the mobile telecommunications market. It is a common platform valid for all sorts of radio access technologies. Primarily focused upon enhancements of packet switched technology, AIPN provides a continued evolution and optimization of the system concept in order to provide competitive edge in terms of both performance and cost. The key benefits of AIPN architecture includes a variety of different access systems' provision, lower costs, universal seamless access, increased user-satisfaction and reduced system latency. But with the advantages of IP come some dangers: as data flow more freely and the internet is open not only to developers but also to all manner of criminals and viruses, developers and operators face new security challenges which should be solved properly. Typical issues for network security and privacy could be how to achieve data confidentiality, integrity and authenticity. The current solutions are the use of cryptographic primitives (such as encryption, signature, and key agreement) or the use of anonymity techniques (such as multiple pseudonyms). However, security and privacy issues considered in a specific network cannot be well solved by directly adopting those techniques. The solutions must take into account the typical characteristics of the network.

Refer Figure 5 in Appendix: Core IP Network, different access systems' provision.

\section{B. VANET Communications}

Vehicular Ad Hoc Networks (VANET) have been envisioned to play an important role in the future wireless service market for safety communications, information and entertainment applications [20]. Examples of safety-oriented applications for VANET are the notifications of emergency situations, such as car accidents or bad weather conditions. Applications in VANET can be implemented by using vehicleto-infrastructure (V2I) communications such as the downloading of music and video files, or vehicle-to-vehicle (V2V) communications, such as in distributed games played among passengers in neighboring vehicles. Challenges of future research in VANET may focus on IP mobility and IPv6 deployment in VANET, capacity and mobility connectivity, mobility models for vehicular networks and data dissemination.

\section{High data rates available at cell edges}

A major challenge of future generation systems is to make the high bit rates available in a larger portion of the cell, especially to users in cell edges and in an exposed position in between several base stations. In current research, this issue is addressed by cellular repeaters and macro-diversity techniques, also known as Group Cooperative Relay (GCR), as well as by Beam Division Multiple Access (BDMA). In spite of the fact that the current OFDMA is appropriate for next generation systems' radio access, BDMA and GCR are also gaining significant attention as effective radio access techniques.

\section{Cooperative Networks}

In the future, services should be delivered seamlessly anytime, anywhere over heterogeneous networks. As an effective solution, cooperative networking has received significant attention recently. In cooperative networking, individual network entities operate in a coordinated way to implement network goals. It spans both the wireless access segment and the backbone information transport and delivery segment.

\section{E. One unified global standard.}

The WiMAX Forum [21] had previously promoted IEEE $802.16 \mathrm{~m}$ [22] as their solution for a true $4 \mathrm{G}$ standards while 3GPP had promoted a similar, but incompatible, 4G standard called LTE Advanced [23]. The 4G Forum would create a single International Telecommunications Union (ITU) standard, world-wide. The incompatible Mobile WiMAX and LTE standards would then evolve into one single interoperable standard called IMT-Advanced [24]. A single IMT-Advanced unified standard will help address bandwidth shortages. Currently, incompatible $4 \mathrm{G}$ standards need to be separated from each other to avoid interference. In addition, incompatible systems increase cost and decrease performance. Until the single IMT-Advanced standard becomes reality, the currently incompatible WiMAX and LTE standards are under heavy research to evolve into a single interoperable NGN standard with 100 Mbps (mobile) and 1 Gbps (fixed) speeds, world-wide.

\section{F. Macro Space-Diversity and Advanced Multi-Cell Coordination}

Macro space-diversity refers to the transmission and/or the reception at multiple geographically separated sites. The aim is to enhance signal quality and reduce interference. Related to this field is the Coordination of Multi-Point transmission and reception (CoMP) [23]. The umbrella of CoMP, comprises many different coordination schemes of very different characteristics: ranging from dynamic inter-cell scheduling coordination (DISC) to joint transmission/reception at multiple sites. In DISC case, CoMP can nearly be seen as an extension of the inter-cell interference coordination. In joint transmission, data is transmitted to a mobile terminal jointly from several sites, thereby not only reducing the interference but also increasing the received power. Similarly, in joint reception the signals received at multiple sites are jointly 
processed for enhanced reception performance. Maximumratio combining and interference-rejection combining are efficient schemes used to combine the uplink transmission received at multiple points. This is, in many respects, similar to softer handover used within a site, for example in WCDMA/HSPA [25] based systems, but extended to multiple sites. Challenges for future research, regarding joint reception and transmission concerns lower latency in the communication between the network node involved in the joint processing and the different antennas involved in the reception/transmission.

\section{G. Direct Device-to-Device Communication}

Direct Device-to-Device (D2D) communication is considered as one possible evolution path to extend NGN' radio-access technology. That is, making it possible for two mobile terminals to communicate directly with each other without going via the network. This has been studied in academia for some years, for example as part of the European research project WINNER [26].Emergency situations and national security and public safety are possible scenarios where the direct D2D communication could be of interest. Moreover, situations where no network infrastructure is available and situations where network infrastructure is available but when communication directly between the terminals could be more efficient are other scenarios for efficient applications of direct D2D communications. Furthermore, an attractive application for D2D communication is the possibility to use NGNs as a radioaccess technology at home. When outside home, the devices can communicate with a cellular network, but while at home they can be connected, even without a SIM card, to the local network covering the home. However, this area is still in its beginning and scenarios where D2D communication offers benefits and how to interact with infrastructure-based communications pose challenges for future research.

\section{H. Global Coverage : Satellites, and High-altitude platforms}

Very high data rates and more service applications could be allowed by the integration of satellites and terrestrial mobile communications. The global coverage systems, achieved by this integration, have been developed by four courtiers. The global position system (GPS) has been developed by USA, the COMPASS system by China, the GALILEO system by EU and the GLONASS by Russia [27]. These independent systems are difficult for space roaming hence a challenge of NGNs is to unite the four systems to get space roaming. At lower altitudes than satellites, high-altitude platforms (HAP) are receiving more and more attention for providing coverage to a large area while staying tens of kilometers above in the air for long periods of time [28]. HAP is a quasi-stationary aircraft; it is specially designed to operate at a very high altitude $(17-22 \mathrm{~km})$ and is able to stay there for days. However, the new generations of HAPs will expand this period to several years! This goal is a challenges for future research. Instead of powering current HAPs by batteries or engines, which need recharging or refueling, solar energy is one of best options currently being used for under trial HAPs. HAP can be a manned or unmanned and is capable to cover a small region much more effectively than satellites because it operates at much lower altitudes. Lower altitude also means much lower power consumption and smaller delay compared to satellites. Furthermore, deploying a satellite drains significant time and monetary resources, in terms of development and launch. HAPs cost much lower than satellite and are rapidly deployable. The applications of HAPs cover the following areas:

- High-speed wireless communications, Research on HAPs is being actively carried largely in Europe to deliver high speed connectivity to users, over areas of up to $400 \mathrm{~km}$. It has gained significant interest because HAPs will be able to deliver data rates similar to a broadband wireless access network (such as WiMAX) while providing a coverage area similar to that of a satellite.

- $\quad$ Surveillance, security and real-time monitoring, one example of a HAP used for surveillance and security is RQ-4 Global Hawk UAV [29]. It possesses a highly sophisticated sensor system and is able to deliver digital sensor data in real-time to a ground station. Powered by a turbofan engine, it can stay in the air for continuous 36 hours. another future use for HAP, currently being under continuous development, is monitoring of a particular region for activities such as flood detection, seismic monitoring, remote sensing and disaster management.

- Weather and environment monitoring, the most common use of HAPs is for measuring environmental changes or to keeping track of weather. Recently, NASA in partnership with The National Oceanic and Atmospheric Administration (NOAA) has started using the aforementioned Global Hawk UAV to study Earth's Atmosphere.

A special form of HAPs is High Altitude Stratospheric Platforms (HASP), which are low cost platforms able to fly at altitudes ranging between 17 and $20 \mathrm{~km}$ for weeks to months. They are attractive for the provision of future personal communication services. The derivation of a sophisticated channel model for the communication link between the platform and terrestrial mobile users or stations constitutes a challenge for future research.

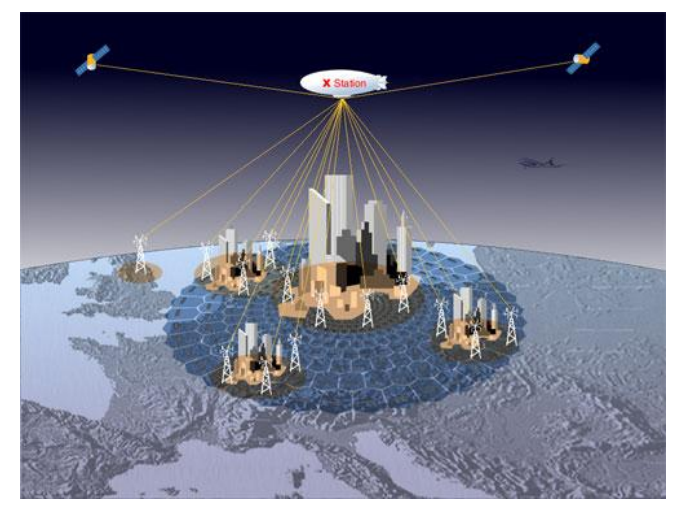

Figure 6: HAP (balloons) linked with various terrestrial and satellite networks. 


\section{DEVELOPING APPLICATIONS}

This section focuses on the most developing applications for NGNs such as World-wide wireless web (WWWW), machine-type communication, wireless sensor networks (WSNs), wearable devices with AI capabilities and EHealthcare systems.

\section{A. World-Wide Wireless Web $(W W W W)$}

WWWW are comprehensive wireless-based web applications that include multimedia capability that are beyond 4G speeds. The wireless Web refers to use of the World Wide Web through a wireless terminal, such as a cellular telephone or personal digital assistant (PDA). WWWW is expected to provide anytime and anywhere connection to e-mail, mobile banking, instant messaging, weather, travel information, and other services. In general, sites aiming to accommodate wireless users must provide services in a format displayable on typically small wireless terminals. WWWW is widely considered as a cornerstone for NGNs.

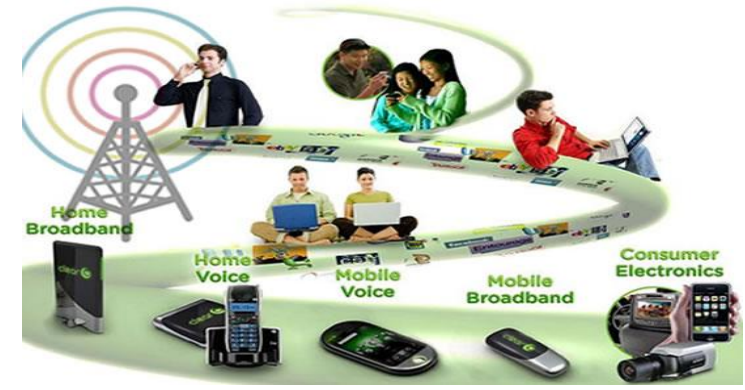

Figure 7: WWWW, comprehensive wireless-based web applications.

\section{B. Machine-Type Communication}

Various types of connectivity has become a realistic option for machine-type communication due to the increased availability of mobile broadband nowadays. Applications of machine-type communication, currently handled by $4 \mathrm{G}$ systems, spans a wide range of applications: from massive deployment of low-cost battery-powered sensors to remotecontrolled utility meters, to surveillance cameras. However, other unlimited applications pose challenges in terms of a vast amount of devices connecting to the network. Handling such a large number of devices, improvements in the area of connection setup and power efficient handling of control signaling in the radio-access network may be of great interest for research and development of NGN.

\section{Sensor Networks}

Wireless sensor networks (WSNs) are designed by grouping mini and/or micro-sized sensors that are equipped with micro-processors, necessary memory and radio transceivers. Sensors are powered by low-energy batteries. WSNs can measure distance, vehicle direction and speed, wind direction and speed, humidity, temperature, chemicals, light, vibrations, seismic data, acoustic data, strain, torque, load, pressure, and other things. WSNs have emerged as a promising solution to a wide range of applications that would redefine the way in which man lives and works. The emergence of nano sensors and nano-enabled sensors have added more applications in many industries, among them transportation, communications, medicine, safety, and security. However, more elaborations of nano sensors for widespread and commercial usage constitute challenges for future research.

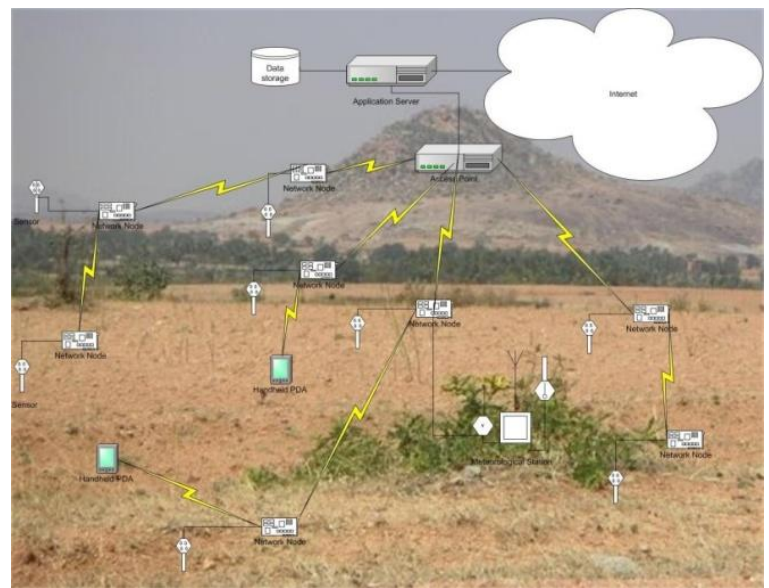

Figure 8: WSNs, a promising solution to a wide range of applications.

\section{Wearable devices with AI capabilities.}

Wearable devices are mini electronic devices, worn by the human being under or on top of clothing and it could be even inserted under skin, Figure 9. They are related to the field of ubiquitous computing and the development of wearable computers. With ubiquitous computing upgraded with AI capability, wearable devices enter our everyday life. They are able to perform multi-tasks without needing to stop what one is doing! They can therefore be an extension of man's mind and/or body. The future development of the wearable devices goes in line with mobile computing, ambient intelligence and ubiquitous computing. Related research challenges include power management, software architectures and connectivity with wireless and personal area networks.

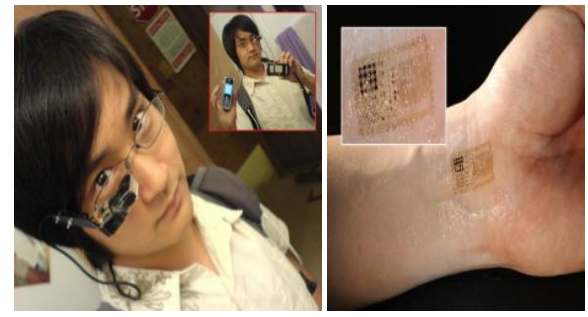

Figure 9: Wearable devices, worn by the bearer under, with or on top of clothing but it could be inserted under skin, source from [30].

\section{E. E-Healthcare Systems}

Future wireless-equipped healthcare systems are expected to offer high quality services to patients through a variety of applications enabled by information and wireless communication technologies. They can remotely and continuously monitor the patients' health status at home and outdoor. Figure 10 shows the aforementioned technologies; wearable devices and wireless sensors integrated with cellular and wireless systems for patient' services. Early detection of patients' emergency situations makes it possible to provide timely first-aid and access to patients' health information in a pervasive manner, thereby improving both system reliability 
and efficiency. The challenges of research and development in the application of wireless communication technologies in healthcare systems include energy-efficiency, Quality-ofServices (QoS), design of healthcare architectures, emergency detection and response, security and privacy, and legal issues.

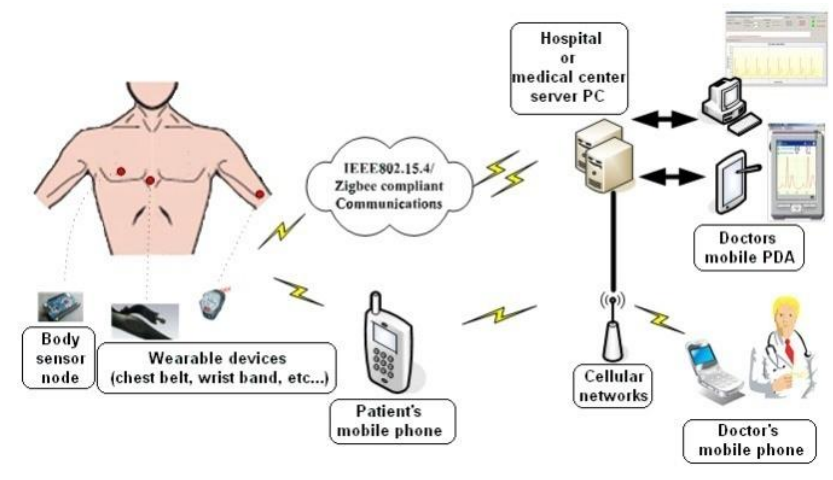

Figure 10: E-healthcare, enabled by wireless and information technologies.

\section{CONCLUSION}

System re-configurability based on cognitive radio and software defined radio constitutes one of the main challenges of future research and development for NGNs. Nanotechnology is expected to revolutionize the mobile telecommunications industry where it has significant impacts on both the terminal and core network. All IP network, direct device-to-device communication and Vehicular Ad Hoc Networks are viable for enhancing network' performance and affordability of NGNs. Solid interworking and adaptive coupling of heterogeneous networks are mandatory until one unified global standard for NGNs becomes reality. Global coverage using satellites and high-altitude platforms are possible evolution paths for space roaming and NGN' radioaccess extension. Finally, machine-type communication, wireless sensor networks, wearable devices and e-healthcare systems will pave the way for the most developing applications of NGNs.

\section{REFERENCES}

[1] Erik Dahlman et al., "4G LTE/LTE-Advanced for Mobile Broadband", Elsevier, 2011

[2] URL: http://www.ipv6.org

[3] S. Haykin, Cognitive radio: Brain-empowered wireless communications, IEEE J. Sel. Area. Comm. 23 (2) (February 2005) 201-220.

[4] Friedrich K. Jondral, "Software-Defined Radio-Basics and Evolution to Cognitive Radio" EURASIP Journal onWireless Communications and Networking 2005:3, 275-283

[5] URL: http://www.sdrforum.org

[6] Leonardo S. Cardoso et al.,"Vandermonde-Subspace Frequency DivisionMultiplexing Receiver Analysis" IEEE 21st International Symposium on, Turkey (2010).

[7] IEEE 802.21: Media Independent Handover,URL: http://www.ieee802.org/21
[8] WWI Ambient Networks.URL: http://www.ambient-networks.org

[9] E. Gustafsson and A. Jonsson, "Always Best Connected," IEEEWireless Communications Magazine, February 2003.

[10] IST-FP6 IP MAGNET (My personal Adaptive GlobalNETwork), URL: http://www.telecom.ece.ntua.gr/magnet

[11] IST-FP6 IP MAGNET Beyond (My personal Adaptive Global

NET and Beyond), URL: http://www.ist-magnet.org

[12] Liljana M. Gavrilovska, Vladimir M. Atanasovski, "Interoperability in Future Wireless Communications Systems: A Roadmap to 4G", Microwave Review, June, 2007.

[13] TRUST homepage: http://www.ist-trust.org

[14] IST-FP5 MOBIVAS (Mobile Value-Added Services), URL: http://mobivas.cnl.di.uoa.gr

[15] IST-FP5 IP PACWOMAN (Power Aware Communications forWirelessOptiMized personal Area Networks),URL: http://www.imec.be/pacwoman

[16] Qiwei Zhang et al. "A Reconfigurable Radio Architecture for Cognitive Radio in Emergency Networks" Proceedings of the 9th European Conference on Wireless Technology

[17] R.K.Jain, Risal Singh, "Role of Nanotechnology in future wireless and communication systems", National seminar proceeding, Academy of Business \& Engineering Science Ghaziabad, pp-19-28, 16-17th January 2009.

[18] Peter Mell and Timothy Grance, "The NIST Definition of Cloud Computing" US National Institute of Standards and Technology Special Publication 800-145, September 2011.

[19] URL: www.cloudcomputingtechnology.org

[20] http://www.vanet.info/

[21] URL: http://www.wimaxforum.org

[22] IEEE 802.16m-07/002r4, TGm System Requirements Document (SRD).

[23] Erik Dahlman et al., "4G LTE/LTE-Advanced for Mobile Broadband", Elsevier, 2011

[24] ITU-R, ITU paves way for next-generation $4 \mathrm{G}$ mobile technologies; ITU-R IMT-advanced $4 \mathrm{G}$ standards to user new era of mobile broadband communications, ITU Press Release, 21 October 2010.

[25] http://www.3gpp.org/release 7

[26] Wireless World Initiative New Radio, Eurescom, 2006, https://www.istwinner.org.

[27] Psiaki, M. L., "Block Acquisition of weak GPS signals in a software receiver", Proceedings of ION GPS 2001, the 14th International Technical Meeting of the Satellite Division of the Institute of Navigation, Salt Lake City, Utah, September 11-14, 2001, pp. 28382850

[28] High Altitude Platforms for Wireless Communications, T.C.Tozer, D. Grace, Electronics\&Communication Engineering Journal June 2001.

[29] http://en.wikipedia.org/wiki/RQ-4_Global_Hawk.

[30] http://www.scienceahead.com/entry/top-21-wearable-technologies/

\section{AUTHORS PROFILE}

Currently working as associate professor at the University of Palestine, Dr. Mousa was granted the $\mathrm{PhD}$ on " $4 \mathrm{G}$ Cellular and WLAN Inter-working Networks" with excellent degree from the National Technical University of Athens, Dept. of Electrical \& Computer Engineering in 2004. Dr. Mousa obtained a D.E.A (equivalent Master degree) in Digital Telecommunication Systems from \{Ecole Nationale Supérieure des Télécommunications/PARIS \} in 1996 and the (B.Sc.) of Electronic Engineering from \{Middle East Technical University/Ankara\} in 1992. 


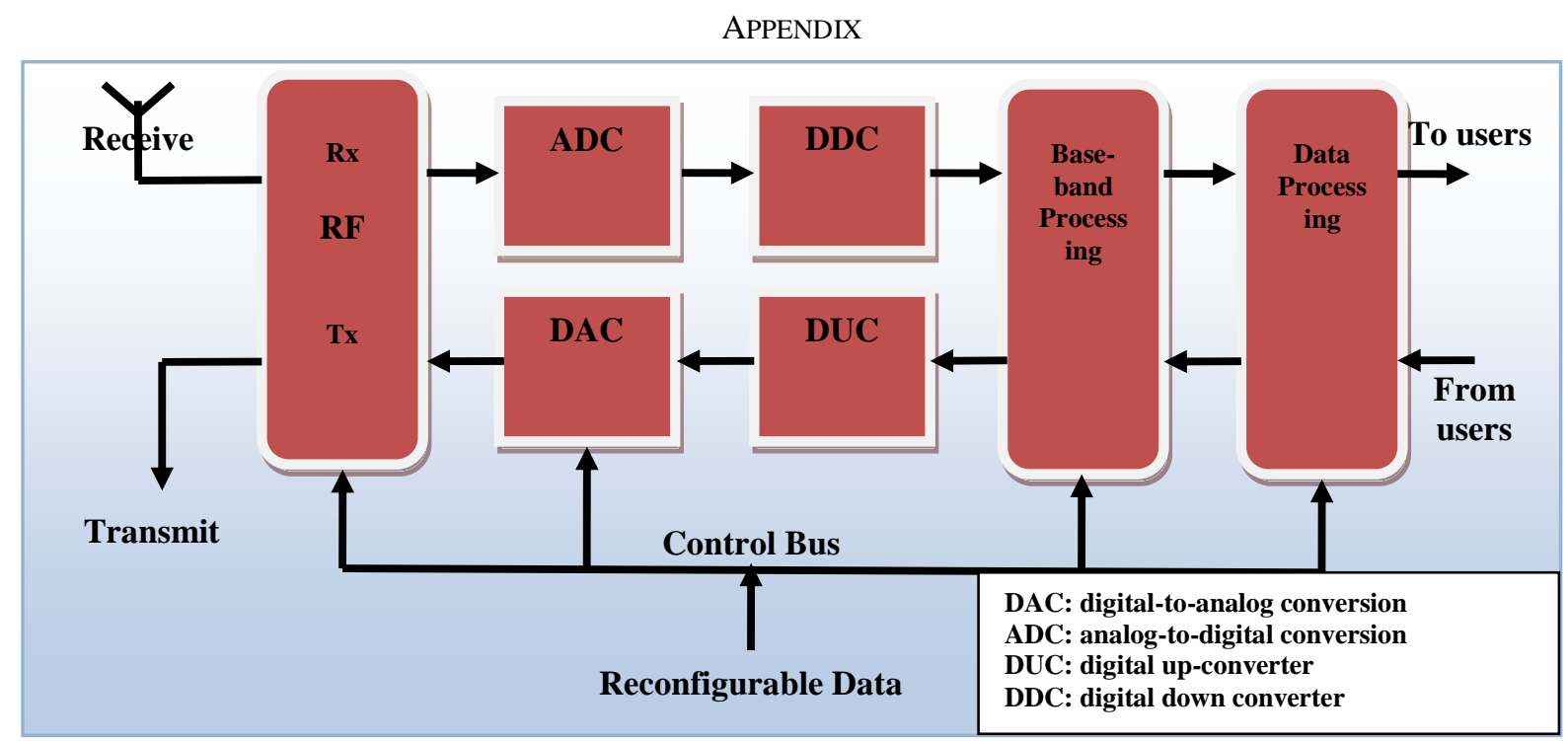

Figure 1: General SDR transceiver

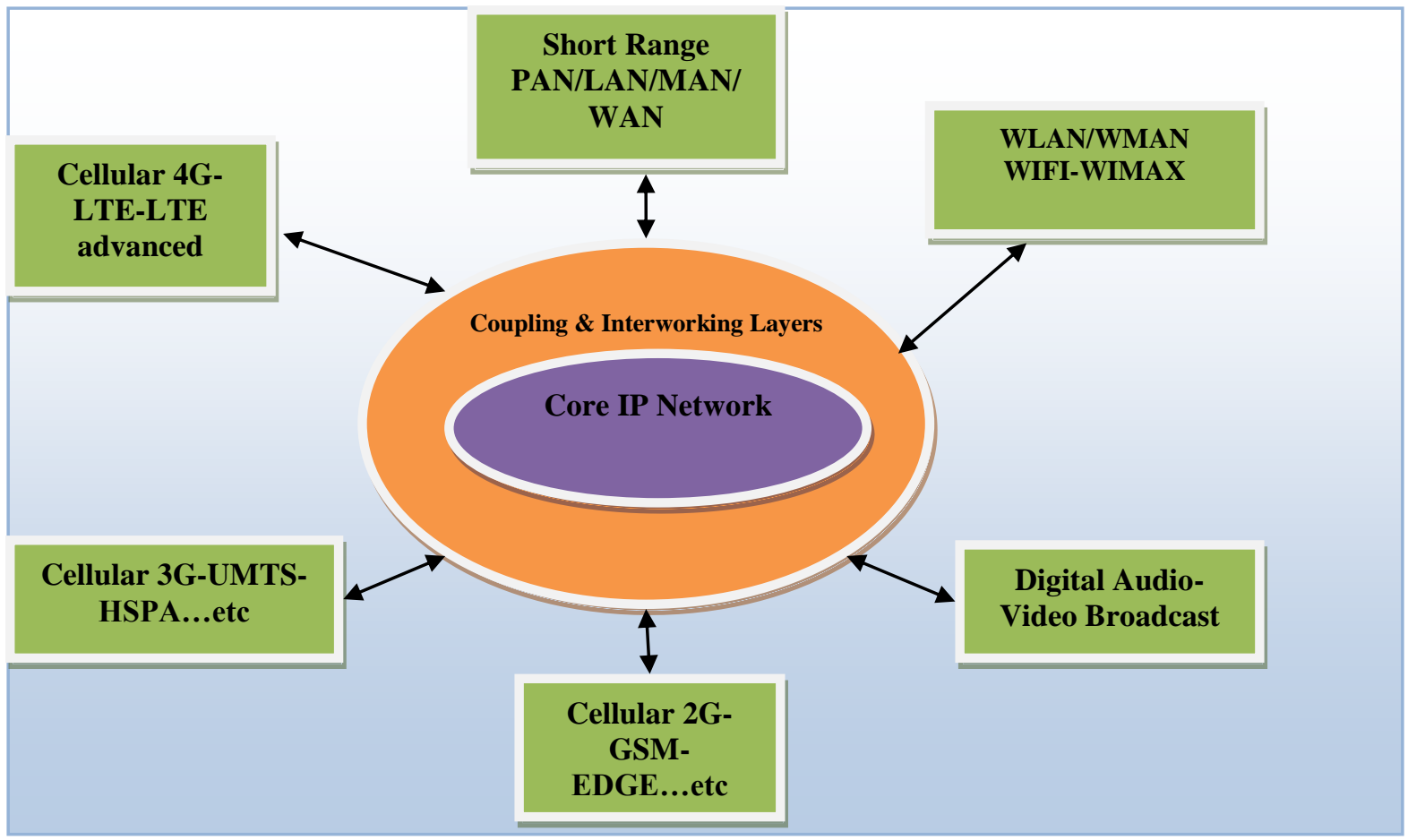

Figure 5: Core IP Network, different access systems’ provision. 